\title{
Characterization of mycobacterial species specificity of 14 separate epitopes which reacted with monoclonal antibodies to the 65,000 molecular weight protein molecule of Mycobacterium leprae
}

\author{
T M BUCHANAN, H NOMAGUCHI, D $\mathrm{C}$ ANDERSON, $\mathrm{R} A$ \\ YOUNG, T P GILLIS, W J BRITTON, J IVANYI, A H J KOLK, O \\ CLOSS, R BLOOM \& V MEHRA \\ University of Washington, Seattle, WA, Whitehead Institute for Bio- \\ medical Research, Cambridge, MA, Gillis W. Long Hansens Disease \\ Center, Carville, LA, Albert Einstein College of Medicine, Bronx, Ny, \\ USA, Osaka University, Osaka, Japan, University of Sydney, Sydney, \\ Australia, Tuberculosis Unit, Hammersmith Hospital, London, Eng- \\ land, Royal Tropical Institute, Amsterdam, The Netherlands, and Na- \\ tional Institute of Public Health, Oslo, Norway
}

This study examined the number of different epitopes recognized by 23 monoclonal antibodies known to recognize the $65 \mathrm{~K}$ dalton protein of the leprosy bacillus, and the mycobacterial species specificity of those 14 monoclonal antibodies shown to recognize different epitopes.

\section{Materials and methods}

MONOCLONAL ANTIBODIES (MOAb)

All of the monoclonal antibodies studied recognized the $65 \mathrm{~K}$ dalton protein band of $M$. leprae as identified by Western Blot (1) or Gel-Immuno-Radio-Assay (2). Antibodies IIC8, IIIC8, IIIE9, IVD8, IIH9, and IVD2 were obtained from Dr. T.M. Buchanan, Seattle, Washington (3-5), antibodies Y1.2, E3.14, T2.3 and C1.3 from Drs. Vijay Mehra and B.R. Bloom, Bronx, New York (3), antibodies SL12, SL14, SL22, SL26, and SL28 from Dr. W.J. Britton, Sydney, Australia (6), antibody ML-30 from Dr. J. Ivanyi, London, England (7), antibody E423 from T. Gillis, Carville, Louisiana (5), antibodies F47-10, F67-2, F67-13, and F67-18 from Dr. Arend H.J. Kolk, Amsterdam, The Netherlands, and antibodies MOC2 and MOC6 from Dr. O. Closs, Oslo, Norway.

Each monoclonal antibody was purified by staphylococcal protein A or sizing chromatography and the purified antibody was radiolabelled with ${ }^{125}$ I using a chloramine $\mathrm{T}$ (8) or Bolton-Hunter (8) procedure. An antigenic preparation of sonicated M. leprcie (9) was coated to Immulon I removawells (Dynatech). The binding of each 125I-labelled antibody to the antigen coated wells was evaluated for inhibition by a 1:100 dilution of ascites fluid of each monoclonal antibody. Monoclonal antibodies with different inhibition patterns from all of the other MOAbs were presumed to recognize different epitopes, and when more than a sin- 
gle antibody recognized a given epitope, a single representative antibody was chosen for further study. In some instances where the radiolabelled antibody reacted poorly with antigen bound to polystyrene plates, the antibody was reacted with the same antigen bound to nitrocellulose. Inhibition of this binding by unlabelled MOAb in a 1:100 dilution was evaluated by autoradiography.

\section{EVALUATION OF MYCOBACTERIAL SPECIES SPECIFICITY}

Bacterial sonicate antigens of each of 23 mycobacterial species were prepared as previously described (9) and adjusted to a $1 \mathrm{mg} / \mathrm{ml}$ protein concentration. The species tested were $M$. bovis $\mathrm{BCG}, M$. bovis, $M$. chelonei, $M$. diernhoferi, $M$. duvali, $M$. flavescens, $M$. fortuitum, $M$. gastri, M. gordonae, M. intracellulare, M. kansasii, M. leprae, M. marinum, M. nonchromogenicum, M. peregrinum, M. phlei, M. scrofulaceum, M. smegmatis, M. terrae, $M$. thamnopheos, M. triviale, M. tuberculosis H37Rv, M. ulcerans, and $M$. vaccae. One microgram protein in five microliters volume was spotted onto nitrocellulose in a template of the 23 species plus a buffer negative control per paper strip and allowed to air dry. The nitrocellulose template strips were blocked at $37^{\circ} \mathrm{C}$ for 1 hour with $5 \%$ nonfat milk in phosphate buffered saline (PBS), and then incubated with a 1:500 - 1:2000 dilution of ascites fluid containing each respective monoclonal antibody in PBS containing $1 \%$ nonfat milk, at room temperature (RT) for 30 minutes. The template strips were drained of antibody and washed x 5 minutes in PBS with gentle shaking at RT, followed by washing x 15 minutes in $0.5 \mathrm{M} \mathrm{NaCI}$ in phosphate buffer containing. $05 \%$ Tween- $\varnothing$, followed by a second 5 minute wash with PBS. The goat anti-mouse immunoglobulin peroxidase conjugate (Cappel) was diluted 1:2000 in $1 \%$ nonfat milk-PBS and incubated with the template strips at RT for 30 minutes. The strips were then drained, and washed at RT with PBS x1 x 5 min, followed by 4 washes of 10 minutes each in $0.5 \mathrm{M} \mathrm{NaCI}$ in PBS with $.05 \%$ Tween 20 , followed by a final wash in PBS x 5 minutes. The washed template strips were reacted for $30-45$ minutes with a $.015 \%$ $\mathrm{H}_{2} \mathrm{O}_{2}$ - color development (4-Cl-I-naphthol, BioRad) solution until the dots developed a purple color. The templates were then washed with distilled water several times and air dried and stored in the dark or in some cases were stored in distilled water prior to air drying and storage. The dot reactions were graded as negative (-) or positive in a range of 1 (low) to 4 (high) intensity.

\section{Results}

Of the 23 MOAbs studied, 14 appeared to recognize different epitopes based upon crosscompetition studies. The distinct MOAbs and other MOAbs which had identical reactivities to those 14 are summarized in Table 1 .

The species specificity of the 14 distinct epitopes recognized is summarized in Table 2. A group of 5 MOAbs represented by IIIE9 recognized an epitope found only on M. leprae, and another antibody (F67-13) recognized all sonicate antigens weakly $(\leqslant 1)$ but gave a $2+$ reaction with $M$. leprae. MOAb ML-30 reacted strongly with only 3 species and the remaining MOAbs reacted with eight or more species. Only MOAb IIC8 reacted with all 23 mycobacterial species tested.

\section{Acknowledgements}

The authors are grateful to the WHO IMMLEP monoclonal antibody bank for providing some of the antibodies used in this study, and to Duane Olsen, Nancy Jenkins, Charlotte Richards and Sigrid McAlister for excellent technical assistance. This study was supported in part by the IMMLEP component of the UNDF/World Bank/WHO Special Program for Research and Training in Tropical Disease, by the Rockefeller foundation Program for Re- 
search on Great Neglected Diseases, by Public health Service Grant AI-22007 and Contract NO1 AI-52582 from the National Institute of Allergy and Infectious Disease, National Institutes of Health, and by a grant from the Japanese component of the US-Japan Collaborative Program in Leprosy for Dr. Nomaguchi.

Table 1. MOAbs which appeared distinct as evaluated by cross-competition studies

$\operatorname{MOA} b(s)$

IIC8

F67-2

IIIC8

ML-30

Y 1.2

IIIE9, IVD8, MOC2,

SL12, SL26

IVD2

C 1.3

IIH9, E3.14, MOC6

E423

SL22

SL28

F67-13

F67-18
MOAbs other than homologous which inhibited

F67-2 (69\%)

IIC8 $(95 \%)$

none

none

none

IVD2 $(67 \%)$ and each of the group of $5 \mathrm{M}$. leprae specific MOAbs listed which all recognized the same epitope IIIE9 $(51 \%)$

E423 (24\%)

IVD2 (22\%)

none

ML-30 (20\%)

none

none

none

Table 2. Species specificity of 14 distinct epitopes recognized* by MOAbs on the 65,000 dalton protein of $\mathrm{M}$. leprae

\section{Epitope (MOabs)}

IIC8

F67-2

IIIC8

ML-30

Y 1.2

IIIE9, IVD8, MOC2,

SL12, SL26

IVD2

C1.3

IIH9, E3.14, MOC6

E423

SL22

\section{Mycobacterial Species** Recognized}

all species

bov GCG, bov, chel, flav, gas, gord, kan,lep, phlei, scrof, smeg, ter, tham, tub, and ulc.

dier, duv, flav, gas, gor, lep, per, scrof, smeg, ter, tham, ulc and vac.

lep, tham, and tub.

bov BOG, chel, duv, flav, gas, gord, int, kan, lep, scrof, ter, tham, tub, and ulc.

lep

bov, chel, duv, flav, gas, gor, lep, scrof, ter, tham, and ulc. bov, flav, gord, kan, lep, scrof, tham, and ulc.

bov BCG, chel, flav, gor, kans, lep, scrof, ter, tham, tub, and ulc.

bov BCG, bov, duv, flav, gas, gor, int, kan, lep, per, scrof, smeg, ter, tub, and ulc.

bov BCG, bov, chel, duv, flav, gas, gor, int, kan, lep, phlei, scrof, smeg, ter, tham, tub, ulc, and vac. 
Epitope (MOabs)

SL28

F67-13

F67-18
Mycobacterial Species** Recognized

bov BCG, chel, dier, duv, flav, fort, gas, gor, int, kan, lep, mar, non, per, ter, tham, triv, tub, and ulc.

lep

bov BCG, bov, chel, duv, flav, gas, gor, int, kan, lep, per, phlei, scrof, smeg, ter, tham, triv, tub, ulc and vac.

$*$ The sonicate antigen preparation from these species of mycobacteria produced a dot reaction of 2-4 intensity (scale $0-4$ ) in dot blot immunoassays with the MOAb indicated.

** bov $\mathrm{BCG}=M$. bovis $\mathrm{BCG}$, bov $=M$. bovis, chel $=M$. chelonei, dier $=M$. diernhoferi, duv $=$ M. duvali, flav $=M$. flavescens, fort $=M$. fortuitum, gas $=M$. gastri, gor $=$ $M$. gordonae, int $=M$. intracellulare, $\mathrm{kan}=M$. kansasii, lep $=M$. leprae, mar $=$ $M$. marinum, non $=M$. nonchromogenicum, per $=M$. perigrinum, phlei $=M$. phlei, scrof $=M$. scrofulaceum, smeg $=M$. smegmatis, ter $=M$. terrae, tham $=$ $M$. thamnopheos, triv $=M$. triviale, tub $=$ tuberculosis, ulc $=M$. ulcerans, vac $=$ M. vaccae

\section{References}

1 Hook E W, D A Olsen, and T M Buchanan. 1984. Analysis of the antigen specificity of the human immune response to complicated gonococcal infection. Infect. Immun. 43:706-709.

2 Poolman, J.T., and T.M. Buchanan. 1984. Monoclonal antibody activity against native and denatured forms of gonococcal outer membrane proteins as detected within ultra thin, longitudinal slices of polyacrylamide gels. J. Immunol. Meth. 75:265-274.

3 Engers, H.D., M. Abe, B.R. Bloom, V. Mehra, W. Britton, T.M. Buchanan, S.K. Khanolkar, D.B. Young, O. Closs, T. Gillis, M. Harboe, J. Ivanyi, A.H.J. Kolk, and C.C. Shepard. 1985. Results of a World Health Organization-sponsored workshop on monoclonal antibodies to Mycobacterium leprae. Infect. Immun. 48:603-605.

4 Gillis, T.P., and T.M. Buchanan. 1982. Monoclonal antibodies to Mycobacterium leprae: Production and partial characterization. Infect. Immun. 37:172-178.

5 Gillis, T.P., R.A. Miller, D.B. Young, S.R. Khanolkar, and T.M. Buchanan. 1985. Immunochemical characterization of a protein associated with the cell wall of Mycobacterium leprae. Infect. Immun. 49:275-281.

6 Britton, W.J., L. Hellqvist, A. Basten, and R.L. Raison. 1985. Mycobacterium leprae antigens involved in human immune responses. I. Identification of four antigens by monoclonal antibodies. J. Immunology 135:4171-4177.

7 Ivanyi, J., S. Sinha, R. Aston, D. Cussel, M. Deen, and U. Sengupta. 1983. Definition of species specific and cross-reactive antigen determinants of Mycobacterium leprae using monoclonal antibodies. Clin. Exp. Immunol. 52:528-536.

8 Bolton, A.E., and W.M. Hunter. 1973. The labelling of proteins to high specific radioactivities by conjugation to a 125 I-containing acylating agent. Application to the radioimmunoassay. Biochem. J. 133:529-539.

9 Young, D.B., M.J. Fohn, S.R. Khanolkar, T.M. Buchanan. 1985. Monoclonal antibodies to a 28,000 molecular wt protein antigen of Mycobacterium leprae. Clin. Exp. Immunol. 60:546-662. 